\title{
Continuum Excitation and Pseudospin Wave in Quantum Spin-Liquid and Quadrupole Ordered States of $\mathbf{T b}_{2+x} \mathbf{T i}_{2-x} \mathrm{O}_{7+y}$
}

\author{
Hiroaki Kadowaki, ${ }^{1}$ Mika Wakita, ${ }^{1}$ Björn Fåk, ${ }^{2}$ Jacques Ollivier, ${ }^{2}$ Seiko \\ Ohira-Kawamura, ${ }^{3}$ Kenji Nakajima, ${ }^{3}$ Hiroshi Takatsu, ${ }^{4}$ and Mototake Tamai ${ }^{1}$ \\ ${ }^{1}$ Department of Physics, Tokyo Metropolitan University, Hachioji-shi, Tokyo 192-0397, Japan \\ ${ }^{2}$ Institute Laue Langevin, BP156, F-38042 Grenoble, France \\ ${ }^{3}$ Neutron Science Section, MLF, J-PARC Center, Shirakata, Tokai, Ibaraki 319-1195, Japan \\ ${ }^{4}$ Department of Energy and Hydrocarbon Chemistry, \\ Graduate School of Engineering, Kyoto University, Kyoto 615-8510, Japan
}

(Dated: September 12, 2018)

\begin{abstract}
The ground states of the frustrated pyrochlore oxide $\mathrm{Tb}_{2+x} \mathrm{Ti}_{2-x} \mathrm{O}_{7+y}$ have been studied by inelastic neutron scattering experiments. Three single-crystal samples are investigated; one shows no phase transition $\left(x=-0.007<x_{\mathrm{c}} \sim-0.0025\right)$, being a putative quantum spin-liquid (QSL), and the other two $(x=0.000,0.003)$ show electric quadrupole ordering (QO) below $T_{\mathrm{c}} \sim 0.5 \mathrm{~K}$. The QSL sample shows continuum excitation spectra with an energy scale $0.1 \mathrm{meV}$ as well as energyresolution-limited (nominally) elastic scattering. As $x$ is increased, pseudospin wave of the QO state emerges from this continuum excitation, which agrees with that of powder samples and consequently verifies good $x$ control for the present single crystal samples.
\end{abstract}

PACS numbers: $75.10 . \mathrm{Kt}, 75.40 . \mathrm{Gb}, 75.70 . \mathrm{Tj}, 78.70 . \mathrm{Nx}$

\section{INTRODUCTION}

Geometrically frustrated magnets have been actively studied in recent years 1. These include classical and quantum spin systems on two-dimensional triangle [2, 3] and kagomé 4, 5, lattices, and three-dimensional pyrochlore-lattice systems [6]. For classical systems, prototypes of which are the trianglar-lattice antiferromagnet [2] and the spin ice [7-9], many investigations have been performed for several decades using a number of theoretical and experimental techniques [1]. Possibilities of quantum spin liquid (QSL) states in frustrated magnets, which date back to the theoretical proposal of the RVB state [10, are recently under hot debate. Highlyentangled many-body wave functions without magnetic long-range order (LRO), anticipated in QSL states, provide theoretically challenging problems [11. Experimentally, finding out real QSL substances, e.g., Refs. 12 16, and investigating QSL states using available techniques, e.g., Refs. [17 21], have been challenging explorations.

A non-Kramers pyrochlore system $\mathrm{Tb}_{2} \mathrm{Ti}_{2} \mathrm{O}_{7}$ (TTO) has attracted much attention since interesting reports of absence of magnetic LRO down to $0.1 \sim 0.4 \mathrm{~K}[22] 24$, which could be interpreted as a QSL candidate [25, 26] or quantum spin ice (QSI) [27, 28. On the other hand, a phase transition at $T_{\mathrm{c}} \sim 0.5 \mathrm{~K}$ detected by a specific heat peak suggesting a hidden LRO [29, 30], seemed to contradict with the QSL interpretation. We resolved this contradiction by showing that ground states of TTO are highly sensitive to off-stoichiometry, i.e., $x$ (and/or $y$ ) of $\mathrm{Tb}_{2+x} \mathrm{Ti}_{2-x} \mathrm{O}_{7+y}$ 31. It was shown that $x$ of powder samples is much easier to control than crystal samples, and that there are two ground states: a hidden LRO $\left(x>x_{\mathrm{c}} \sim-0.0025\right)$ state and a QSL $\left(x<x_{\mathrm{c}}\right)$ state 31.

By carefully analyzing experimental data of TTO sam- ples, we proposed an effective pseudospin-1/2 Hamiltonian for a typical TTO sample with the LRO groud state, $\mathrm{Tb}_{2.005} \mathrm{Ti}_{1.995} \mathrm{O}_{7+y}\left(T_{\mathrm{c}} \sim 0.5 \mathrm{~K}\right)[3235$. This Hamiltonian consists of interaction terms among magnetic dipole moments and those among electric quadrupole moments 36. 37. It naturally explains that the hidden LRO is an electric quadrupole order (QO) 36, 37, and that a neighboring phase to this QO phase is a theoretically proposed U(1) QSL state [28, 38, possibly occurring in TTO samples without LRO 32 .

Therefore, the long-standing question of "what is the QSL state of TTO?" [25, 39] is now about to be addressed using well-controlled single-crystals [40. In other words, one needs to pickup useful clues for solving this question from many experimental results reported to date, e.g., Refs. [22, 25, 29, 41,63, which could suffer from the off-stoichiometry and its inhomogeneity problems to a certain extent. Although the sample stoichiometry does not necessarily affect all experimental facts, one should cautiously reconsider results of these references. In particular, it should be noted that our evaluation of the sample stoichiometry and those of Refs. 62 64 likely do not coincide.

In this work, we have prepared large single-crystal samples of $\mathrm{Tb}_{2+x} \mathrm{Ti}_{2-x} \mathrm{O}_{7+y}$ with controlled $x$ ( $y$ is determined by the oxidation condition) 40, and performed inelastic neutron scattering (INS) experiments. The resulting INS spectra of TTO samples with and without $T_{\mathrm{c}}$ show that small variation of $x$ really induces a continuous change of INS spectra at low temperatures, reflecting the QSL and QO ground states. This agrees with our previous results of INS on powder samples [31, 32. 


\section{EXPERIMENTAL METHODS}

Polycrystalline samples of $\mathrm{Tb}_{2+x} \mathrm{Ti}_{2-x} \mathrm{O}_{7+y}$ were prepared by the standard solid-state reaction [31]. The two starting materials, $\mathrm{Tb}_{4} \mathrm{O}_{7}$ and $\mathrm{TiO}_{2}$, were heated in air at $1350{ }^{\circ} \mathrm{C}$ for several days with periodic grindings to ensure a complete reaction. The value of $x$ was adjusted by changing the mass ratio of the two materials. Single crystal $\mathrm{Tb}_{2+x} \mathrm{Ti}_{2-x} \mathrm{O}_{7+y}$ rods were grown by the floating zone (FZ) technique from feed rods of sintered powder samples with $-0.04<x<-0.002$ [40. Crystal growth was carried out in an Ar gas flow atmosphere using a double ellipsoidal image furnace (NEC SC-N35HD). They were post-annealed for $2-7$ days at $1000{ }^{\circ} \mathrm{C}$ in air. The $y$ value was determined by this oxidizing condition, where an unmeasurably small increase of $y$ occurred. The offstoichiometry parameter $x$ was evaluated by measuring the lattice parameter $a(T, x)$ using a high-resolution Xray diffractometer (RIGAKU SmartLab) equipped with a $\mathrm{Cu} \mathrm{K} \mathrm{K}_{\alpha 1}$ monochromator [40. Details of this evaluation are described in the appendix.

For INS experiments we chose seven 15-20 mm crystal rods with small concentration gradient cut from longer crystal rods with $\sim 2 \mathrm{~mm}$ in diameter, and assembled three samples. One sample is $x=-0.007\left(<x_{\mathrm{c}}\right.$, in the QSL range) and consists of three $15 \mathrm{~mm}$ crystal rods with $1.2 \mathrm{~g}$ in total weight. In the inset photograph of Fig. 4 we show one $15 \mathrm{~mm}$ crystal of this QSL sample. To evaluate $x$ of this crystal, two samples $(\sim 1 \mathrm{~mm})$ for the X-ray measurement were cut from two neighbors being very close to the both ends. The resulting lattice parameters ensure that $x=-0.007 \pm 0.002$. Another crystal sample for INS is $x=0.000 \pm 0.002\left(>x_{\mathrm{c}}\right.$, in the QO range) and consists of three $15 \mathrm{~mm}$ crystals with $1.1 \mathrm{~g}$ in total weight. These QSL and QO samples are co-aligned within 1.5 degrees. The third crystal sample is a $20 \mathrm{~mm}$ rod with $x=0.003 \pm 0.002$, which is in the QO range. It is $0.6 \mathrm{~g}$ in weight.

Neutron scattering experiments for the $x=-0.007$ and 0.000 crystal samples were carried out on the time-offlight (TOF) spectrometer IN5 65 operated with $\lambda=8$ $\AA$ at ILL. The energy resolution of this condition was $\Delta E=0.021 \mathrm{meV}(\mathrm{FWHM})$ at the elastic position. The previous INS experiments [31, 32] using the powder samples $(x=-0.005$ and 0.005$)$ were carried out on IN5 operated with $\lambda=10 \AA$. The energy resolution of this condition was $\Delta E=0.012 \mathrm{meV}$ (FWHM) at the elastic position. INS experiments for the $x=0.003$ crystal sample were performed on the TOF spectrometer AMATERAS 66 operated with $\lambda=7 \AA$ at J-PARC. The energy resolution of this condition was $\Delta E=0.024 \mathrm{meV}$ (FWHM) at the elastic position. Each crystal sample was mounted in a dilution refrigerator so as to coincide its $(h, h, l)$ plane with the horizontal scattering plane of the spectrometer. The observed intensity data of the crystal samples were converted to $S(\boldsymbol{Q}, E)$ using Lamp 67. or Utsusemi 68, and further corrected for absorption using a home-made program 69. Construction of
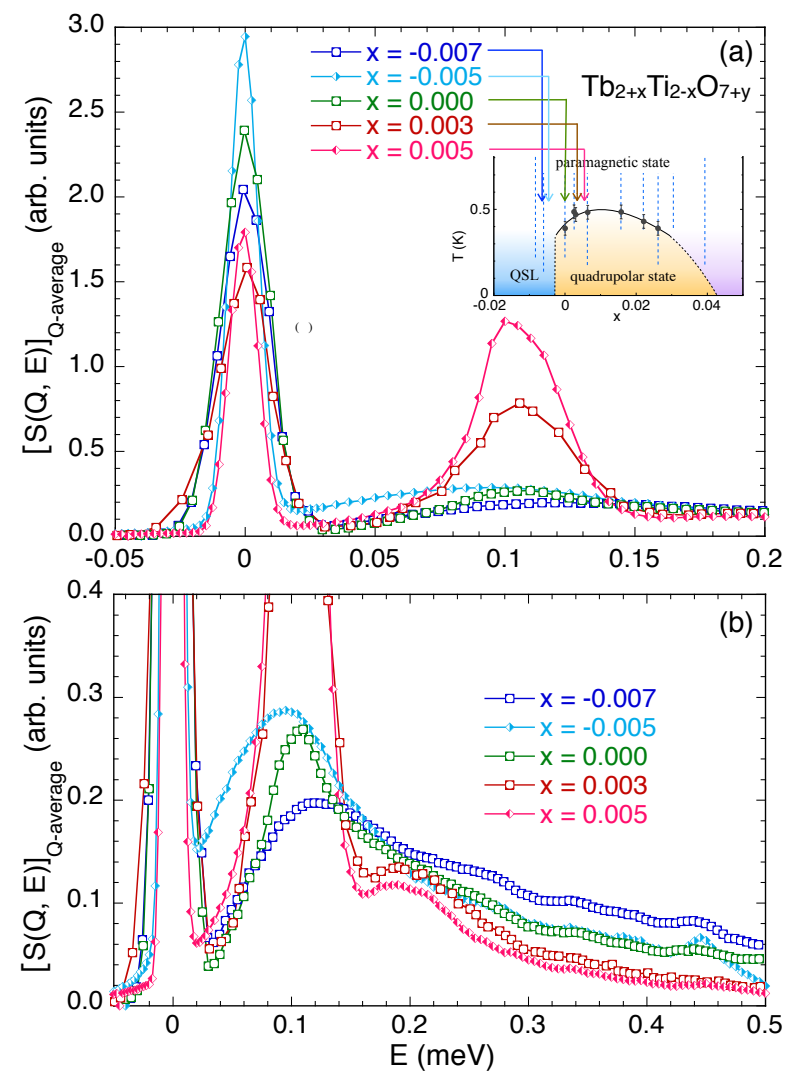

FIG. 1. (Color online) Energy spectra $S(\boldsymbol{Q}, E)$ averaged in a wide $\boldsymbol{Q}$-range, $\boldsymbol{Q}=(h+k, h-k, l)$ with $0<h<0.9$, $0.75<l<1.75,-0.25<k<0.25$ (r.l.u), at $T=0.1 \mathrm{~K}$ for $x=-0.007,0.000$, and 0.003 crystal samples. Two scales are used to show (a) a low energy part and (b) a low intensity part. These spectra are plotted with previous results of powder samples with $x=-0.005$ and 0.005 taken at $T=0.1$ $\mathrm{K}$ 31, 32. The energy spectra of the powder samples are averaged in $0.3<|\boldsymbol{Q}|<0.9 \AA^{-1}$. In the inset of (a), these samples are shown by arrows in the $x-T$ phase diagram of Ref. 40]. The intensity scales of the five samples are approximately normalized as described in the text.

four dimensional $S(\boldsymbol{Q}, E)$ data object from a set of the TOF data taken by rotating each crystal sample are performed using HORACE [70].

Background scattering of $S(\boldsymbol{Q}, E)$ from sample holders etc. in the elastic channel was subtracted. Background in the inelastic channel, which is very small at least in $E<0$ at $T=0.1 \mathrm{~K}$, was neglected. To compare $S(\boldsymbol{Q}, E)$ of the crystal samples we normalize $S(\boldsymbol{Q}, E)$ using a relation $\int S(\boldsymbol{Q}, E) d \boldsymbol{Q} d E=$ const, where $\boldsymbol{Q}$ integrations extend to $|\boldsymbol{Q}|<\infty$ and $E$ is integrated in a range which covers all scattering contribution from the ground state doublet of the crystal field. This exact relation at $T=0$ is approximated by that with $T=0.1 \mathrm{~K}$ and over a wide integration range: an $E$ range of $-0.1<E<0.5 \mathrm{meV}$, and a $\boldsymbol{Q}$ range of $\boldsymbol{Q}=(h+k, h-k, l)$ with $0<h<0.9$, $0.75<l<1.75$, and $-0.25<k<0.25$ (r.l.u). This approximation is probably good for the present three crystal 

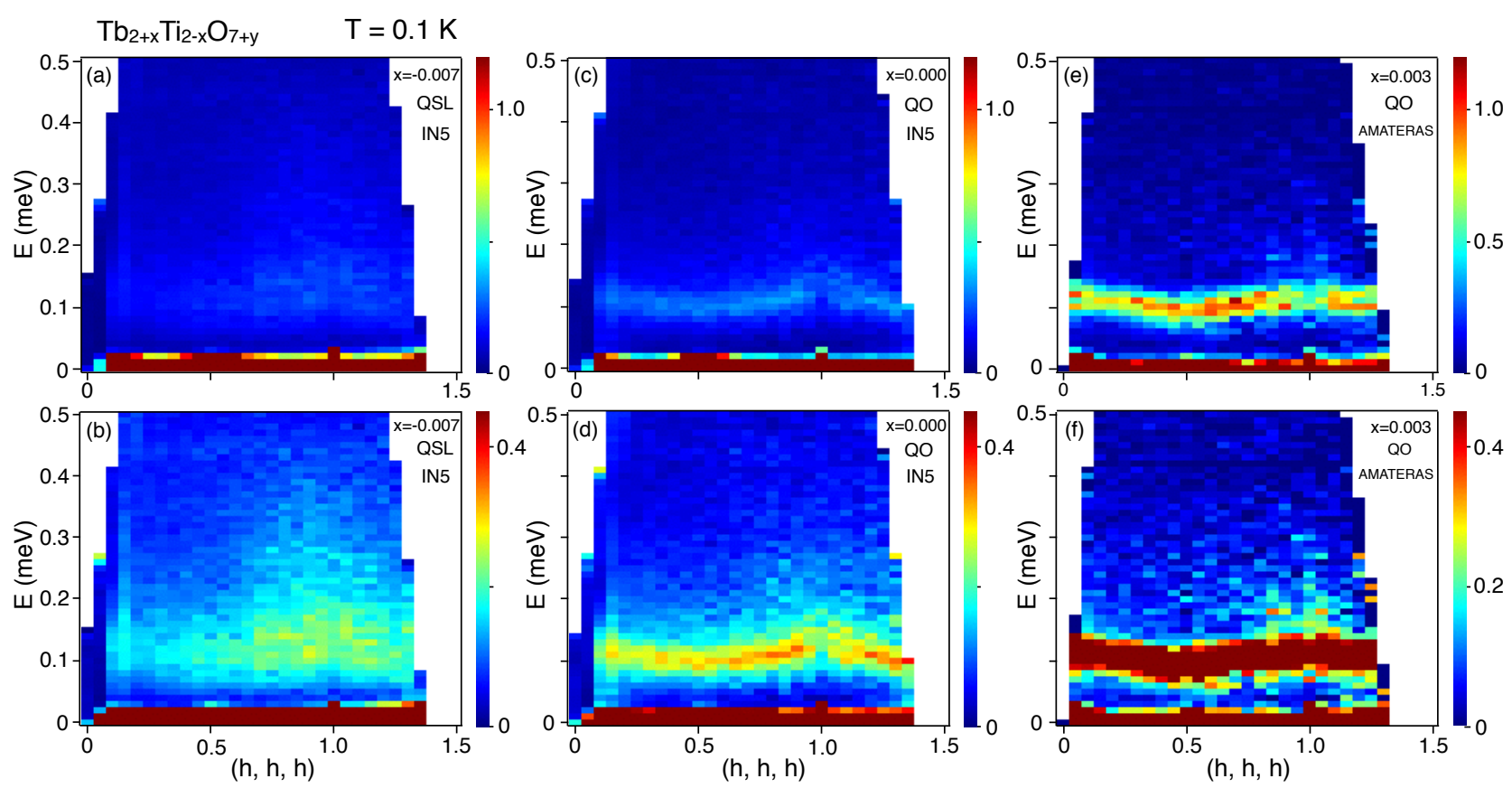

FIG. 2. (Color online) $Q-E$ slices along a $[1,1,1]$ direction measured at $T=0.1 \mathrm{~K}$. The color bars show linear intensity scales of $S(\boldsymbol{Q}, E)$ in the "arb. units" used in Fig. 1. (a,b) show $S(\boldsymbol{Q}, E)$ of the QSL sample with $x=-0.007$. (c,d) and (e,f) show $S(\boldsymbol{Q}, E)$ of the QO samples with $x=0.000$ and 0.003 , respectively. Two colormaps of (a,c,e) and (b,d,f) are used for high and low intensity ranges, respectively.

samples. To compare these data with $\int S(\boldsymbol{Q}, E) d \boldsymbol{Q}$ of the powder samples [31, 32], we used the same relation, where the integration range was replaced to $0.3<|\boldsymbol{Q}|<0.9$ $\AA^{-1}$ and $-0.1<E<0.5 \mathrm{meV}$.

\section{RESULTS AND DISCUSSION}

\section{Inelastic Neutron Scattering: Q-Averaged Spectra}

In Fig. 1 we show $\boldsymbol{Q}$-averaged $E$-scans ( $E$-cuts) of $S(\boldsymbol{Q}, E)$ at $0.1 \mathrm{~K}$ for the crystal samples. In this figure, our previous results using the powder samples with $x=-0.005$ and 0.005 taken at $0.1 \mathrm{~K}$ 31, 32 are also plotted for comparison. These data of the powder samples are $\boldsymbol{Q}$-averaged $S(\boldsymbol{Q}, E)$ which are averaged in $0.3<|\boldsymbol{Q}|<0.9 \AA^{-1}$. To clearly show $x$ dependence of these $E$-cuts we approximately normalized the scattering intensities. By the present definition, the energy integra-

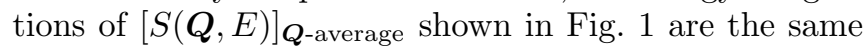
for the five samples. These normalized "arb. units" are used in the subsequent figures.

One can see from Fig. 1 that energy spectra of the two QSL samples with $x=-0.007$ and -0.005 show overall similarities. For the $x=-0.005$ sample, which is closer to the quantum phase transition, an increase of spectral weight at low energies $(0<E<0.06 \mathrm{meV})$ are seen. As $x$ is increased to $x=0.000$, which is in the $\mathrm{QO}$ range, the spectra are qualitatively changed; a small peak around $E=0.1 \mathrm{meV}$ appears in the $E$-cut. This excitation peak develops as $x$ is further increased to $x=0.003$ and 0.005 . We note that the energy spectra of the crystal and the powder samples show reasonable $x$ dependence, despite the fact that the crystal and powder samples were prepared in very different ways.

The energy spectra obviously consist of two parts: energy-resolution-limited (nominally) elastic scattering and inelastic scattering. We note that the existence of these two scatterings have been commonly observed in INS experiments of all TTO samples, e.g. 23], although $\boldsymbol{Q}$ - and $E$-dependence of $S(\boldsymbol{Q}, E)$ problematically depended on the sample quality. The energy-resolution limited scattering in the present experimental condition implies that two-spin time correlations $\left\langle S_{i}^{z}(0) S_{j}^{z}(t)\right\rangle$ have slowly decaying parts in a time scale much longer than $66 \mathrm{ps}(E \sim 0.01 \mathrm{meV}, 2.4 \mathrm{GHz}$, or $0.1 \mathrm{~K})$. On the other hand, the inelastic scattering with the energy scale 0.1 meV implies that $\left\langle S_{i}^{z}(0) S_{j}^{z}(t)\right\rangle$ has a short time scale of 6.6 ps.

\section{Inelastic Neutron Scattering: Q-Dependent Spectra}

Fig.2 2 shows typical $\boldsymbol{Q}$-dependence of the inelastic scattering at $T=0.1 \mathrm{~K}$ by plotting $Q-E$ slices along a $[1,1,1]$ direction. For closer inspection of energy spectra, $E$-cuts at $\boldsymbol{Q}=(1 / 2,1 / 2,1 / 2)$ and $(1,1,1)$ are plotted in Fig. 3 . One can see from these figures that the QSL sample shows gapless continuum excitation spectra [Figs. 2(a) 

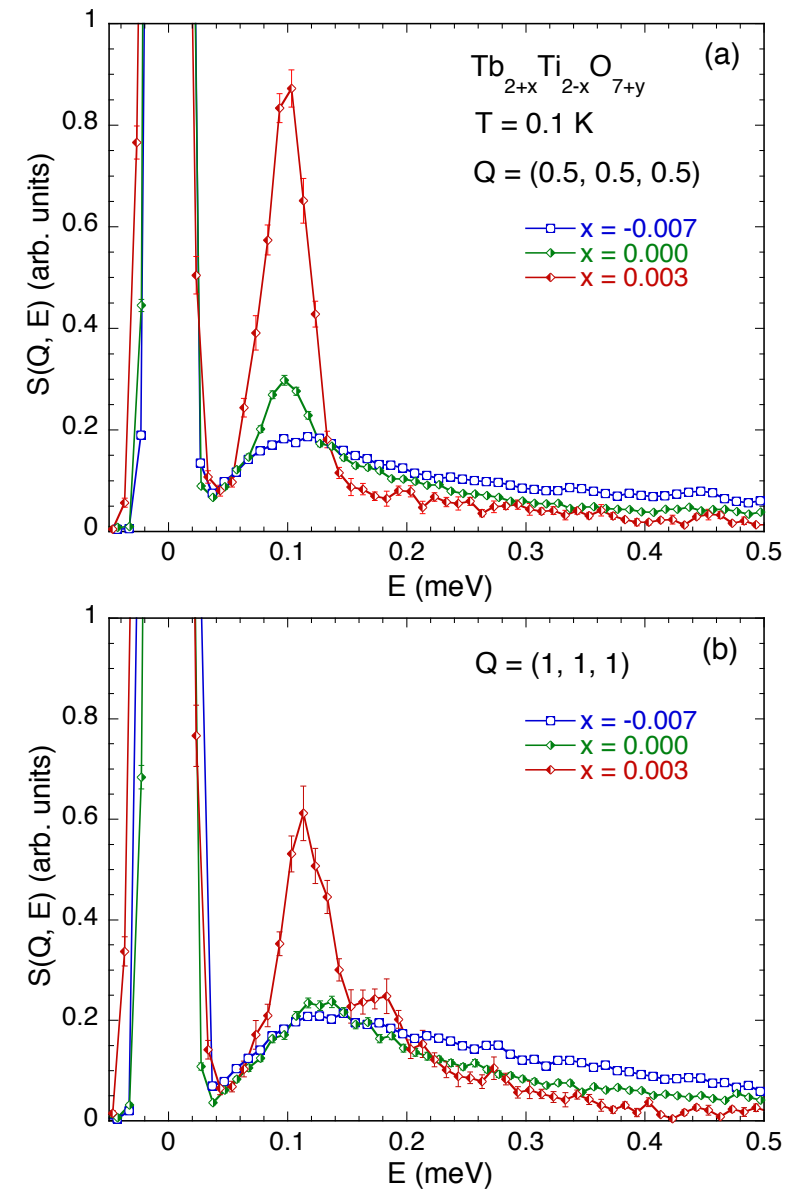

FIG. 3. (Color online) Inelastic energy spectra $S(\boldsymbol{Q}, E)$ at $T=0.1 \mathrm{~K}$ of the three samples are shown for (a) $\boldsymbol{Q}=$ $(1 / 2,1 / 2,1 / 2)$ and (b) $\boldsymbol{Q}=(1,1,1)$. The "arb. units" of Fig. 1 are used.

and (b)]. These continuum excitations may possibly be those of "magnetic monopole" [28, 71] (or vison [39]) of the U(1) QSL state. On the other hand, for the QO sample with $x=0.000$ [Figs. 2(c) and (d)] gapped pseudospin wave excitation with flat dispersion relation emerges from the continuum excitation. This pseudospin wave excitation becomes more distinct for the QO sample with $x=0.003$ [Figs. 2(e) and (f)]. We also searched $S(\boldsymbol{Q}, E)$ for the propagating excitations reported in Ref. [46, although nothing similar was found in the present data.

The pseudospin wave excitation can be understood as composite wave of magnetic-dipole and electricquadrupole moments as discussed in Refs. 32, 33. We note that this excitation bears resemblance to spinorbital excitations in $d$-electron systems 72 75. Starting from the proposed model parameter 32 , we are now performing refinements of interaction parameters to reproduce the observed dispersion relation of the $x=0.003$ sample. In addition to this pseudospin wave, there remains the continuum excitation in Figs. 2(e) and (f).
Especially, the E-cut at $\boldsymbol{Q}=(1,1,1)$ [Fig. 3(b)] shows that the continuum excitation $(E>0.15 \mathrm{meV})$ is substantially strong. These facts suggest that the QO state has large zero-point quantum fluctuations.

Finally, let us make a comment on a simple question of "what the off-stoichiometry $x$ does?" in relation to the phase diagram of Fig. 1(a). A possible answer to this question or a scenario is as follows. In the $x=0$ sample, there is a tiny amount of site exchange between non-magnetic $\mathrm{Ti}^{4+}$ and magnetic $\mathrm{Tb}^{3+}$ sites. As a consequence, there remains randomness effects in the nominally stoichiometric sample. As $x$ is increased from $x=0$, more magnetic $\mathrm{Tb}^{3+}$ or $\mathrm{Tb}^{4+}$ ions occupy the $\mathrm{Ti}^{4+}$ site. These magnetic ions reduce the effective magnetic interactions, while quadrupole interactions are very weakly affected. For small $x>0$, the QO state is observed. By further increasing $x$ the randomness effects on quadrupole interactions become stronger and the quadrupole LRO disappears $(x>0.04)$. On the other hand, as $x$ is decreased from $x=0$, magnetic $\mathrm{Tb}^{3+}$ ions residing on the $\mathrm{Ti}^{4+}$ site are removed. For $x<-0.0025$ the effective magnetic interactions become sufficiently strong and the QSL state is observed. Recently randomness effects on QSL states are studied theoretically [76 80] and experimentally $81-83$. In these studies, much larger model disorders than those in TTO $(-0.01<x<0.005)$ are studied.

\section{CONCLUSIONS}

The ground states of the frustrated pyrochlore oxide $\mathrm{Tb}_{2+x} \mathrm{Ti}_{2-x} \mathrm{O}_{7+y}$ have been studied by inelastic neutron scattering experiments using three single-crystal samples: one putative QSL sample with $x=-0.007\left(<x_{\mathrm{c}}\right)$ and two QO samples with $x=0.000$ and 0.003 . Small concentration gradient $(|\Delta x|<0.002)$ of these samples has enabled us to observe $x$-dependence of inelastic excitation spectra at low temperatures. The QSL sample shows continuum excitation spectra with an energy scale $0.1 \mathrm{meV}$ as well as energy-resolution-limited (nominally) elastic scattering. As $x$ is increased, pseudospin wave of the QO state emerges from the continuum excitation.

\section{ACKNOWLEDGMENTS}

This work was supported by JSPS KAKENHI grant numbers 25400345 and 26400336. The neutron scattering performed using ILL IN5 (France) was transferred from JRR-3M HER (proposal 11567, 15545) with the approval of ISSP, Univ. of Tokyo, and JAEA, Tokai, Japan. The neutron scattering experiments at J-PARC AMATERAS were carried out under a research project number 2016 A0327. 


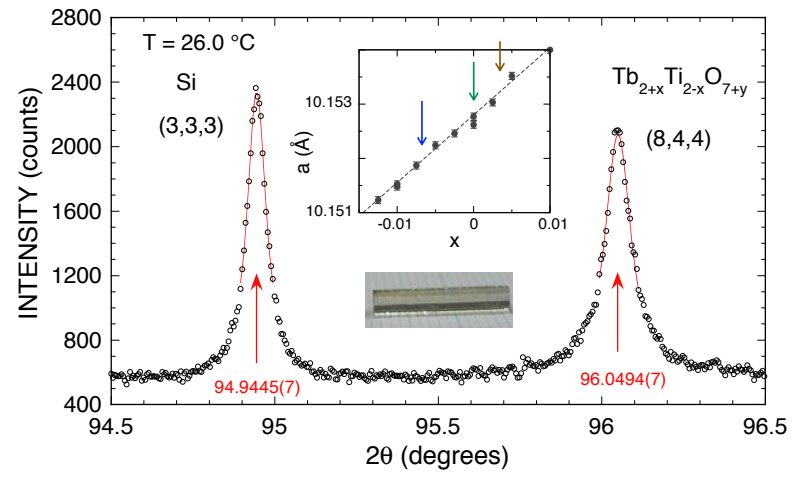

FIG. 4. (Color online) A typical $\theta-2 \theta$ scan of a powder mixture of $\mathrm{Si}$ and crushed-crystalline $\mathrm{Tb}_{2+x} \mathrm{Ti}_{2-x} \mathrm{O}_{7+y}$. The inset plot shows the lattice parameter $a\left(T=26.0^{\circ} \mathrm{C}, x\right)$ of Ref. [40], where three arrows indicate the three samples for the present single-crystal INS experiments. The inset photograph shows a $15 \mathrm{~mm}$ crystal rod, one of the QSL multi-crystal sample.

\section{APPENDIX*}

The off-stoichiometry parameters $(x)$ of $\mathrm{Tb}_{2+x} \mathrm{Ti}_{2-x} \mathrm{O}_{7+y}$ samples were evaluated by measuring the lattice parameter of small crystals $(\sim 1$ $\mathrm{mm})$ cut from single-crystal rods [40. We performed high-resolution $\theta-2 \theta$ scans on powder mixtures $(\sim 5$ $\mathrm{mg}$ ) of polycrystalline $\mathrm{Si}$ and crushed-crystalline $\mathrm{Tb}_{2+x} \mathrm{Ti}_{2-x} \mathrm{O}_{7+y}$ using the X-ray diffractometer. In these measurements, temperature of the mixture was controlled at $T=26.0 \pm 0.1^{\circ} \mathrm{C}$ to minimize experimental error. We determined $x$ using the relation $a\left(T=26.0^{\circ} \mathrm{C}, x\right)=0.124418 x+10.15280$ [40], which was measured using powder samples [31.

Fig. 4 shows a typical $\theta-2 \theta$ scan carried out to mea- sure $a$ and to evaluate $x$ of a small crystal sample. It should be noted that although there are only two peaks of Si 333 and TTO 844 reflections in the scan range $[94.5<2 \theta<96.5$ (deg)], HEIDENHAIN absolute angle encoders installed in the SmartLab ensure high reproducibility of the result. This reproducibility is seen in Fig. 3 of Ref. 84] and Fig. 6 of Ref. 85. In order to further reduce slight systematic errors of $2 \theta$, which are brought about possibly by occasional optical alignments, we repeated the same $\theta-2 \theta$ scan on a standard sample and made a correction. This standard sample was a mixture of Si and the $x=-0.0075$ TTO powder [31]. Thus, we note that our evaluation of the lattice parameter depends on both this standard sample and the certified lattice parameter $a=5.43123(8) \AA$ of Si (NIST SRM640d) 86]. In addition, we note that the oxidizing condition determining $y$ also affects the $x$ evaluation.

We would like to finally mention another problem of the TTO preparation caused by the starting material $\mathrm{Tb}_{4} \mathrm{O}_{7}$. Since $\mathrm{Tb}_{4} \mathrm{O}_{7}$ is not a pure chemical substance, it should be expressed probably as $\mathrm{Tb}_{4} \mathrm{O}_{7+\delta}$ with small $\delta$. This $\delta$, normally unknown, depends on details of the production process of each chemical company. The starting material $\mathrm{Tb}_{4} \mathrm{O}_{7}$ used in our investigations from 2012, i.e., Refs. [31 35, 40] and the preset study, was from a single batch produced by Shin-Etsu Chemical.

Because of these technical problems, one has to be very cautious to compare evaluations of the composition $x$ or/and $y$ of TTO samples by different investigation groups and even by the same group. We think that our samples are self-consistent among Refs. [31,35, 40] and the present study. The sample used in Ref. [30, however, shows slight breaking of this self-consistency, which is seen in Fig. 2 of Ref. 31. Thus we think that it is not sensible to make comments here on samples of other investigation groups.
[1] C. Lacroix, P. Mendels, and F. Mila, eds., Introduction to Frustrated Magnetism (Springer, Berlin, Heidelberg, 2011).

[2] G. H. Wannier, Phys. Rev. 79, 357 (1950)

[3] M. Mekata, J. Phys. Soc. Jpn. 42, 76 (1977)

[4] I. Syôzi, Prog. Theor. Phys. 6, 306 (1951).

[5] M. P. Shores, E. A. Nytko, B. M. Bartlett, and D. G. Nocera, J. Am. Chem. Soc. 127, 13462 (2005).

[6] J. S. Gardner, M. J. P. Gingras, and J. E. Greedan, Rev. Mod. Phys. 82, 53 (2010)

[7] S. T. Bramwell and M. J. P. Gingras, Science 294, 1495 (2001).

[8] C. Castelnovo, R. Moessner, and S. L. Sondhi, Nature 451, 42 (2008)

[9] H. Kadowaki, N. Doi, Y. Aoki, Y. Tabata, T. J. Sato, J. W. Lynn, K. Matsuhira, and Z. Hiroi, J. Phys. Soc. Jpn. 78, 103706 (2009)

[10] P. W. Anderson, Mater. Res. Bull. 8, 153 (1973).

[11] L. Savary and L. Balents, Rep. Prog. Phys. 80, 016502 (2017).
[12] K. Hirakawa, H. Kadowaki, and K. Ubukoshi, J. Phys. Soc. Jpn. 54, 3526 (1985).

[13] Y. Shimizu, K. Miyagawa, K. Kanoda, M. Maesato, and G. Saito, Phys. Rev. Lett. 91, 107001 (2003)

[14] S. Nakatsuji, Y. Machida, Y. Maeno, T. Tayama, T. Sakakibara, J. v. Duijn, L. Balicas, J. N. Millican, R. T. Macaluso, and J. Y. Chan, Phys. Rev. Lett. 96, 087204 (2006)

[15] Y. Li, H. Liao, Z. Zhang, S. Li, F. Jin, L. Ling, L. Zhang, Y. Zou, L. Pi, Z. Yang, J. Wang, Z. Wu, and Q. Zhang, Scientific Reports 5, 16419 (2015)

[16] R. Sibille, E. Lhotel, M. C. Hatnean, G. Balakrishnan, B. Fåk, N. Gauthier, T. Fennell, and M. Kenzelmann, Phys. Rev. B 94, 024436 (2016).

[17] T.-H. Han, J. S. Helton, S. Chu, D. G. Nocera, J. A. Rodriguez-Rivera, C. Broholm, and Y. S. Lee, Nature (London) 492, 406 (2012)

[18] K. A. Ross, L. Savary, B. D. Gaulin, and L. Balents, Phys. Rev. X 1, 021002 (2011)

[19] L.-J. Chang, S. Onoda, Y. Su, Y.-J. Kao, K.-D. Tsuei, 
Y. Yasui, K. Kakurai, and M. R. Lees, Nature Communications 3, 992 (2012).

[20] Y. Shen, Y.-D. Li, H. Wo, Y. Li, S. Shen, B. Pan, Q. Wang, H. C. Walker, P. Steffens, M. Boehm, Y. Hao, D. L. Quintero-Castro, L. W. Harriger, M. D. Frontzek, L. Hao, S. Meng, Q. Zhang, G. Chen, and J. Zhao, Nature (London) 540, 559 (2016).

[21] B. Fåk, S. Bieri, E. Canévet, L. Messio, C. Payen, M. Viaud, C. Guillot-Deudon, C. Darie, J. Ollivier, and P. Mendels, Phys. Rev. B 95, 060402 (2017)

[22] J. S. Gardner, S. R. Dunsiger, B. D. Gaulin, M. J. P. Gingras, J. E. Greedan, R. F. Kiefl, M. D. Lumsden, W. A. MacFarlane, N. P. Raju, J. E. Sonier, I. Swainson, and Z. Tun, Phys. Rev. Lett. 82, 1012 (1999)

[23] Y. Yasui, M. Kanada, M. Ito, H. Harashina, M. Sato, H. Okumura, K. Kakurai, and H. Kadowaki, J. Phys. Soc. Jpn. 71, 599 (2002).

[24] J. S. Gardner, A. Keren, G. Ehlers, C. Stock, E. Segal, J. M. Roper, B. Fåk, M. B. Stone, P. R. Hammar, D. H. Reich, and B. D. Gaulin, Phys. Rev. B 68, 180401 (2003)

[25] M. J. P. Gingras, B. C. den Hertog, M. Faucher, J. S. Gardner, S. R. Dunsiger, L. J. Chang, B. D. Gaulin, N. P. Raju, and J. E. Greedan, Phys. Rev. B 62, 6496 (2000).

[26] Y.-J. Kao, M. Enjalran, A. Del Maestro, H. R. Molavian, and M. J. P. Gingras, Phys. Rev. B 68, 172407 (2003).

[27] H. R. Molavian, M. J. P. Gingras, and B. Canals, Phys. Rev. Lett. 98, 157204 (2007).

[28] M. Hermele, M. P. A. Fisher, and L. Balents, Phys. Rev. B 69, 064404 (2004)

[29] N. Hamaguchi, T. Matsushita, N. Wada, Y. Yasui, and M. Sato, Phys. Rev. B 69, 132413 (2004).

[30] H. Takatsu, H. Kadowaki, T. J. Sato, J. W. Lynn, Y. Tabata, T. Yamazaki, and K. Matsuhira, J. Phys. Condens. Matter 24, 052201 (2012)

[31] T. Taniguchi, H. Kadowaki, H. Takatsu, B. Fåk, J. Ollivier, T. Yamazaki, T. J. Sato, H. Yoshizawa, Y. Shimura, T. Sakakibara, T. Hong, K. Goto, L. R. Yaraskavitch, and J. B. Kycia, Phys. Rev. B 87, 060408 (2013)

[32] H. Takatsu, S. Onoda, S. Kittaka, A. Kasahara, Y. Kono, T. Sakakibara, Y. Kato, B. Fåk, J. Ollivier, J. W. Lynn, T. Taniguchi, M. Wakita, and H. Kadowaki, Phys. Rev. Lett. 116, 217201 (2016).

[33] H. Kadowaki, H. Takatsu, T. Taniguchi, B. Fåk, and J. Ollivier, SPIN 5, 1540003 (2015).

[34] H. Takatsu, T. Taniguchi, S. Kittaka, T. Sakakibara, and H. Kadowaki, J. Phys.: Conf. Series 683, 012022 (2016)

[35] H. Takatsu, T. Taniguchi, S. Kittaka, T. Sakakibara, and H. Kadowaki, J. Phys.: Conf. Series 828, 012007 (2017)

[36] S. Onoda and Y. Tanaka, Phys. Rev. Lett. 105, 047201 (2010)

[37] S. Onoda and Y. Tanaka, Phys. Rev. B 83, 094411 (2011)

[38] S. Lee, S. Onoda, and L. Balents, Phys. Rev. B 86, $104412(2012)$

[39] M. J. P. Gingras and P. A. McClarty, Rep. Prog. Phys. 77, 056501 (2014).

[40] M. Wakita, T. Taniguchi, H. Edamoto, H. Takatsu, and H. Kadowaki, J. Phys.: Conf. Series 683, 012023 (2016).

[41] I. Mirebeau, P. Bonville, and M. Hennion, Phys. Rev. B 76, 184436 (2007)

[42] A. Bertin, Y. Chapuis, P. Dalmas de Réotier, and A. Yaouanc, J. Phys. Condens. Matter 24, 256003 (2012).
[43] Y. Chapuis, A. Yaouanc, P. Dalmas de Réotier, C. Marin, S. Vanishri, S. H. Curnoe, C. Vâju, and A. Forget, Phys. Rev. B 82, 100402 (2010).

[44] A. Yaouanc, P. Dalmas de Réotier, Y. Chapuis, C. Marin, S. Vanishri, D. Aoki, B. Fåk, L.-P. Regnault, C. Buisson, A. Amato, C. Baines, and A. D. Hillier, Phys. Rev. B 84, 184403 (2011).

[45] E. Lhotel, C. Paulsen, P. D. de Réotier, A. Yaouanc, C. Marin, and S. Vanishri, Phys. Rev. B 86, 020410 (2012)

[46] S. Guitteny, J. Robert, P. Bonville, J. Ollivier, C. Decorse, P. Steffens, M. Boehm, H. Mutka, I. Mirebeau, and S. Petit, Phys. Rev. Lett. 111, 087201 (2013)

[47] P. Bonville, I. Mirebeau, A. Gukasov, S. Petit, and J. Robert, Phys. Rev. B 84, 184409 (2011).

[48] S. Petit, P. Bonville, J. Robert, C. Decorse, and I. Mirebeau, Phys. Rev. B 86, 174403 (2012).

[49] P. Bonville, A. Gukasov, I. Mirebeau, and S. Petit, Phys. Rev. B 89, 085115 (2014).

[50] T. Fennell, M. Kenzelmann, B. Roessli, M. K. Haas, and R. J. Cava, Phys. Rev. Lett. 109, 017201 (2012)

[51] T. Fennell, M. Kenzelmann, B. Roessli, H. Mutka, J. Ollivier, M. Ruminy, U. Stuhr, O. Zaharko, L. Bovo, A. Cervellino, M. K. Haas, and R. J. Cava, Phys. Rev. Lett. 112, 017203 (2014)

[52] B. D. Gaulin, J. S. Gardner, P. A. McClarty, and M. J. P. Gingras, Phys. Rev. B 84, 140402 (2011).

[53] K. Fritsch, K. A. Ross, Y. Qiu, J. R. D. Copley, T. Guidi, R. I. Bewley, H. A. Dabkowska, and B. D. Gaulin, Phys. Rev. B 87, 094410 (2013).

[54] K. Fritsch, E. Kermarrec, K. A. Ross, Y. Qiu, J. R. D. Copley, D. Pomaranski, J. B. Kycia, H. A. Dabkowska, and B. D. Gaulin, Phys. Rev. B 90, 014429 (2014).

[55] J. Zhang, K. Fritsch, Z. Hao, B. V. Bagheri, M. J. P. Gingras, G. E. Granroth, P. Jiramongkolchai, R. J. Cava, and B. D. Gaulin, Phys. Rev. B 89, 134410 (2014).

[56] A. J. Princep, H. C. Walker, D. T. Adroja, D. Prabhakaran, and A. T. Boothroyd, Phys. Rev. B 91, 224430 (2015)

[57] L. Bovo, C. M. Rouleau, D. Prabhakaran, and S. T. Bramwell, Nanotechnology 28, 055708 (2017)

[58] M. Hirschberger, J. W. Krizan, R. J. Cava, and N. P. Ong, Science 348, 106 (2015).

[59] M. Ruminy, M. N. n. Valdez, B. Wehinger, A. Bosak, D. T. Adroja, U. Stuhr, K. Iida, K. Kamazawa, E. Pomjakushina, D. Prabakharan, M. K. Haas, L. Bovo, D. Sheptyakov, A. Cervellino, R. J. Cava, M. Kenzelmann, N. A. Spaldin, and T. Fennell, Phys. Rev. B 93, $214308(2016)$

[60] M. Ruminy, F. Groitl, T. Keller, and T. Fennell, Phys. Rev. B 94, 174406 (2016).

[61] E. Constable, R. Ballou, J. Robert, C. Decorse, J.-B. Brubach, P. Roy, E. Lhotel, L. Del-Rey, V. Simonet, S. Petit, and S. deBrion, Phys. Rev. B 95, 020415 (2017)

[62] E. Kermarrec, D. D. Maharaj, J. Gaudet, K. Fritsch, D. Pomaranski, J. B. Kycia, Y. Qiu, J. R. D. Copley, M. M. P. Couchman, A. O. R. Morningstar, H. A. Dabkowska, and B. D. Gaulin, Phys. Rev. B 92, 245114 (2015)

[63] M. Ruminy, L. Bovo, E. Pomjakushina, M. K. Haas, U. Stuhr, A. Cervellino, R. J. Cava, M. Kenzelmann, and T. Fennell, Phys. Rev. B 93, 144407 (2016).

[64] S. Guitteny, I. Mirebeau, P. Dalmas de Réotier, C. V. Colin, P. Bonville, F. Porcher, B. Grenier, C. Decorse, 
and S. Petit, Phys. Rev. B 92, 144412 (2015)

[65] J. Ollivier and H. Mutka, J. Phys. Soc. Jpn. 80, SB003 (2011).

[66] K. Nakajima, S. Ohira-Kawamura, T. Kikuchi, M. Nakamura, R. Kajimoto, Y. Inamura, N. Takahashi, K. Aizawa, K. Suzuya, K. Shibata, T. Nakatani, K. Soyama, R. Maruyama, H. Tanaka, W. Kambara, T. Iwahashi, Y. Itoh, T. Osakabe, S. Wakimoto, K. Kakurai, F. Maekawa, M. Harada, K. Oikawa, R. E. Lechner, F. Mezei, and M. Arai, J. Phys. Soc. Jpn. 80, SB028 (2011).

[67] D. Richard, M. Ferrand, and G. J. Kearley, J. Neutron Research 4, 33 (1996)

[68] Y. Inamura, T. Nakatani, J. Suzuki, and T. Otomo, J. Phys. Soc. Jpn. 82, SA031 (2013).

[69] H. Kadowaki, https://github.com/kadowakih/AbsorptionFactorIN5; https://github.com/kadowakih/AbsorptionFactorAMATERAS.

[70] R. Ewings, A. Buts, M. Le, J. van Duijn, I. Bustinduy, and T. Perring, Nucl. Instrum. Methods Phys. Res. Sect. A 834, 132 (2016)

[71] G. Chen, Phys. Rev. B 96, 195127 (2017)

[72] G. Khaliullin, Prog. Theor. Phys. Suppl. 160, 155 (2005).

[73] K. Tomiyasu and S. Itoh, J. Phys. Soc. Jpn. 75, 084708 (2006)

[74] L. M. Helme, A. T. Boothroyd, R. Coldea, D. Prabhakaran, C. D. Frost, D. A. Keen, L. P. Regnault, P. G. Freeman, M. Enderle, and J. Kulda, Phys. Rev. B 80, 134414 (2009)

[75] L. Das, F. Forte, R. Fittipaldi, C. G. Fatuzzo, V. Granata, O. Ivashko, M. Horio, F. Schindler, M. Dantz, Y. Tseng, D. E. McNally, H. M. Rønnow,
W. Wan, N. B. Christensen, J. Pelliciari, P. OlaldeVelasco, N. Kikugawa, T. Neupert, A. Vecchione, T. Schmitt, M. Cuoco, and J. Chang, Phys. Rev. X 8, 011048 (2018)

[76] K. Watanabe, H. Kawamura, H. Nakano, and T. Sakai, J. Phys. Soc. Jpn. 83, 034714 (2014).

[77] K. Uematsu and H. Kawamura, J. Phys. Soc. Jpn. 86, 044704 (2017)

[78] L. Savary and L. Balents, Phys. Rev. Lett. 118, 087203 (2017).

[79] O. Benton, arXiv:1706.09238.

[80] Z. Zhu, P. A. Maksimov, S. R. White, and A. L. Chernyshev, Phys. Rev. Lett. 119, 157201 (2017)

[81] J.-J. Wen, S. M. Koohpayeh, K. A. Ross, B. A. Trump, T. M. McQueen, K. Kimura, S. Nakatsuji, Y. Qiu, D. M. Pajerowski, J. R. D. Copley, and C. L. Broholm, Phys. Rev. Lett. 118, 107206 (2017)

[82] N. Martin, P. Bonville, E. Lhotel, S. Guitteny, A. Wildes, C. Decorse, M. Ciomaga Hatnean, G. Balakrishnan, I. Mirebeau, and S. Petit, Phys. Rev. X 7, 041028 (2017)

[83] R. Sibille, E. Lhotel, M. Ciomaga Hatnean, G. J. Nilsen, G. Ehlers, A. Cervellino, E. Ressouche, M. Frontzek, O. Zaharko, V. Pomjakushin, U. Stuhr, H. C. Walker, D. T. Adroja, H. Luetkens, C. Baines, A. Amato, G. Balakrishnan, T. Fennell, and M. Kenzelmann, Nature Communications 8, 892 (2017)

[84] K. Goto, H. Takatsu, T. Taniguchi, and H. Kadowaki, J. Phys. Soc. Jpn. 81, 015001 (2012).

[85] H. Takatsu, K. Watanabe, K. Goto, and H. Kadowaki, Phys. Rev. B 90, 235110 (2014).

[86] D. L. Kaiser and R. L. Watters, (2010), NIST Certificate of SRM 640d. 